\title{
La comunidad LGBT+ en el Perú: los retos para incluirla y reconocer sus derechos en el siglo XXI
}

\author{
Marcial Giovanni GutiérRez Lucar ${ }^{(*)}$
}

Abogado y Máster en Derecho por la Universidad de Lima. Estudios de posgrado por la Universidad de Ginebra y el Geneva Center for International

Dispute Settlement.

Profesor de Derecho Civil y Procesal Civil de la Universidad de Lima. Miembro Asociado de ADV Editores - Revista ADVOCATUS.

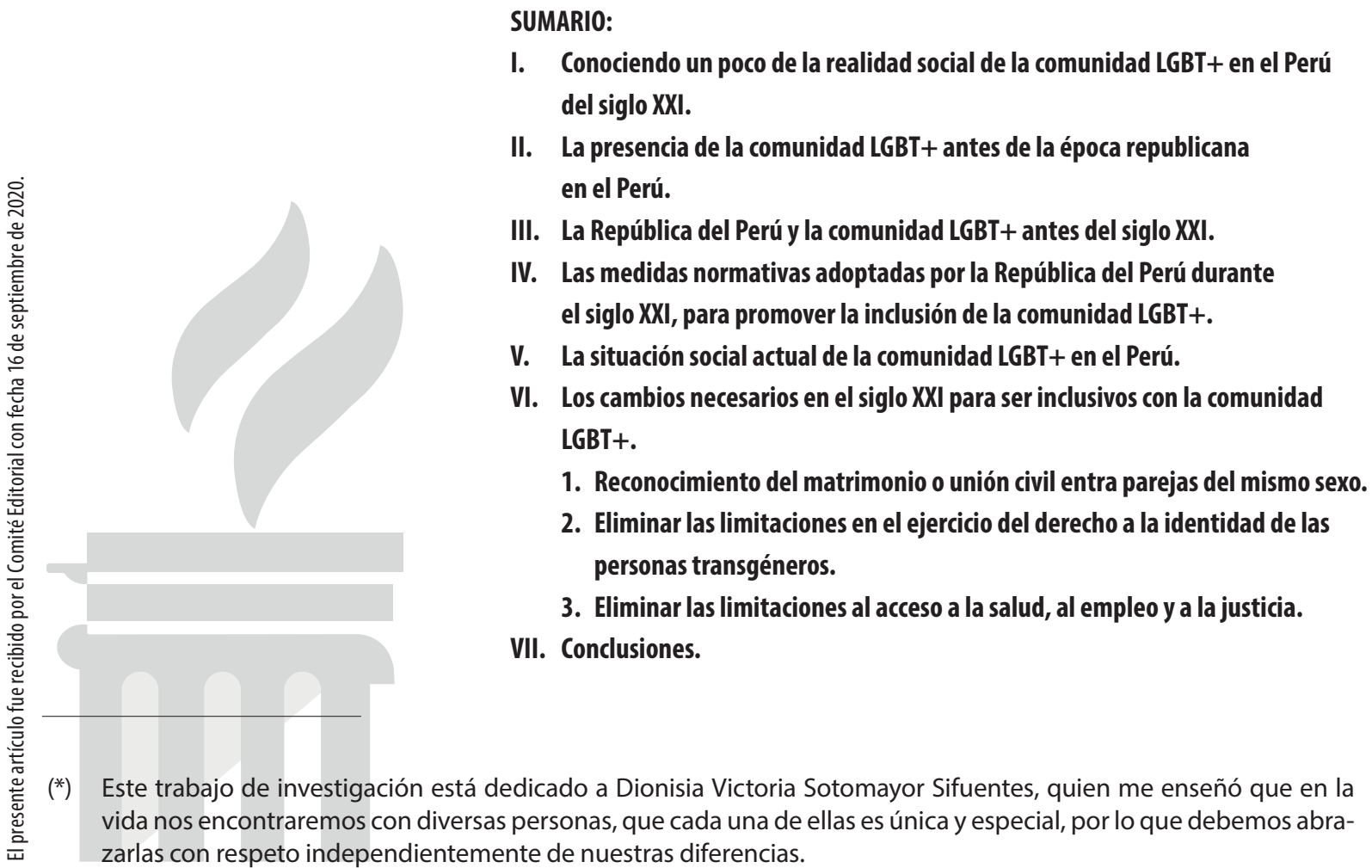




\title{
RESUMEN:
}

El presente artículo abordará la problemática que atraviesan los miembros de la comunidad LGBT+ en el Perú del siglo XXI, desde una perspectiva social como jurídica. Lo que permitirá identificar los cambios normativos que son necesarios de regular, a fin de alcanzar una sociedad más inclusiva y libre de discriminación de las minorías.

Palabras clave: comunidad, discriminación, constitucional, identidad, orientación.

\begin{abstract}
:
This article will address the problems faced by the members of the LGBT+ community in the 21st century in Peru, from a social and legal perspective. This will allow the identification of the legislative changes that need to be regulated in order to achieve a society which is more inclusive and free from discrimination against minorities.
\end{abstract}

Keywords: community, discrimination, constitutional, identity, orientation.

"Todos tenemos dos elecciones: estar llenos de miedo o llenos de amor."

Albert Einstein

\section{CONOCIENDO UN POCO DE LA REALIDAD SOCIAL DE LA COMUNIDAD LGBT+ EN EL PERÚ DEL SIGLO XXI}

Adrián ${ }^{1}$ era un niño que a una temprana edad descubrió que tenía una cualidad especial, ya que, a pesar de haber nacido varón, él no se identificaba como tal. Ahí fue cuando descubrió que, en realidad, era una persona transgénero².

Con el pasar de los años, y como parte de su autodescubrimiento sexual, Adrián decidió que al alcanzar la mayoría de edad iniciaría el procedimiento médico de reasignación de sexo, para que sea identificado físicamente como una mujer. Desde que comenzó su transición, Adrián dejó de existir y dio paso al nacimiento de su verdadero yo: Adriana.
Adriana se encontró ante diversas adversidades, como no contar con un procedimiento que le permita modificar su documento de identidad y, por lo tanto, que su nueva apariencia física carezca de reconocimiento legal. Ello generó que, la discrepancia entre su aspecto de mujer y los papeles que la identificaban como hombre, le vayan cerrando las oportunidades para conseguir un trabajo formal. Así, la única manera que Adriana encontró para subsistir fue la práctica de la prostitución.

La madrugada del 28 de octubre de 2007, después de haber culminado con sus labores, Adriana esperaba un transporte que la lleve a su casa. Mientras el tiempo transcurría, 5 sujetos aparecieron, la agredieron física y psicológicamente, hasta casi dejarla inconsciente. Cuando logró escapar, se acercó a las autoridades policiales para solicitar respaldo, pero, lamentablemente, obtuvo por respuesta una negativa: "soluciona tu problema en otro lado, no te vamos a ayudar".

1. Los hechos relatados se sustentan en las vivencias de Yefri Peña Tuanama, víctima de la transfobia peruana.

«Yefri Peña - Tortura», Incidencia Internacional PROMSEX, acceso el 18 de septiembre de 2020, https://incidenciainternacional.promsex.org/yefri-pena-tortura/.

2. Transgénero: abreviado como "trans", describe a una persona cuya identidad de género no corresponde al sexo biológico. Hace referencia a un rango de identidades que incluye a niños y hombres transgénero: personas que se identificaban como niño u hombre, pero que fueron asignadas como mujer al nacer; niñas y mujeres transgénero: personas que se identifican como niña o mujer, pero que fueron asignadas como hombres al nacer."

National Geographic, «Género, La Revolución», National Geographic en español, Volumen 40, n.1 (2017): 13. 
A los pocos minutos de que le negaron la ayu$\mathrm{da}$, al frente de la misma estación policial, Adriana se encontró nuevamente con sus agresores, quienes volvieron a atacarla, salvo que en esta oportunidad le generaron 20 cortes profundos en la espalda, le desfiguraron el rostro y la dejaron en paz cuando pareció que estaba muerta. Pese a que los policías vieron lo ocurrido, ninguno de ellos acudió en su auxilio.

Otras personas trasladaron a Adriana al hospital más cercano; sin embargo, pese a su crítico estado, las autoridades del nosocomio no la atendieron ya que su identificación no concordaba con su aspecto físico. Adriana terminó en su casa, donde ella y su madre limpiaron sus heridas. Días después, debido a la falta de atención médica, Adriana agravó y entró en estado de coma por poco más 30 días.

Adriana sobrevivió al ataque descrito y, actualmente, participa como voluntaria en las campañas sobre salud sexual y derechos humanos realizadas por el Ministerio de Salud, educando a sus ex compañeras de trabajo. Además, es fundadora de la casa trans de Lima Este y lucha por el reconocimiento de los derechos de las personas transgénero en el Perú.

Los hechos narrados ponen sobre la mesa la realidad que viven las personas transgéneros en el Perú, de la cual no son ajenas los demás integrantes $^{3 \text { y }}{ }^{4}$ de la comunidad LGBT+ -lesbiana, gay, bisexual, transgénero y otros- peruana.
La situación descrita nos lleva a consultarnos ¿en dónde nos encontramos actualmente, respecto a los derechos de la comunidad LGBT+ en el Perú? Y, además, ¿qué esperamos que ocurra sobre ello a lo largo del siglo XXI? Estas son las interrogantes que absolveremos a lo largo del presente artículo.

\section{LA PRESENCIA DE LA COMUNIDAD LGBT+ ANTES DE LA ÉPOCA REPUBLICANA EN EL PERÚ}

Cuando el debate vinculado al reconocimiento de derechos de la comunidad LGBT+ ha estado sobre la mesa en los últimos años, diversas autoridades han señalado que son ideas que van en contra de la naturaleza humana y, además, que no son propias a la sociedad peruana.

En ese sentido, queremos aprovechar esta publicación para realizar las siguientes precisiones:

a) En primer lugar, no debemos olvidar que el ser humano u homo sapiens es una especie del género de los primates, es decir, que pertenece a la familia de los homínidos. Solo que cuenta con algunas habilidades más desarrolladas, como las sociales.

En dicho sentido, al igual que en otras especies de animales, podemos señalar que la homosexualidad es un hecho innato y cuyas manifestaciones pueden ser encontradas a lo largo de la propia naturaleza. Por ejemplo ${ }^{5}$, podemos refe-

3. Un niño de 12 años se suicidó en lquitos dado que su padrastro lo humilló, cortándole el pelo, por enterarse que era gay. Asimismo, sus compañeros de escuela lo habían acosado y humillado, empeorando la situación que vivía.

«Un Niño de 12 Años Se Suicida En Perú, Después de Que Su Padrastro Castigara Por Ser Gay"», Ilga-lac, acceso el 18 de septiembre de 2020, https://www.ilga-lac.org/2015/02/05/un-nino-de-12-anos-se-suicida-en-peru-despues-deque-su-padrastro-castigara-por-ser-gay/.

4. Caso Pilar Fachín, quien fue agredida por el hermano de su enamorada. Él la atacó con un machete, logrando romperle el maxilar, rebanarle la oreja y fracturarle el cráneo. Adicionalmente, le dejó cicatrices en las manos, brazos y espalda.

«Pilar Fachín - Crimen Por Lesbofobia», Incidencia Internacional PROMSEX, acceso el 18 de septiembre de 2020, https://incidenciainternacional.promsex.org/pilar-fachin-crimen-lesbofobia/.

5. Gabriel J. Martín, Quiérete mucho, maricón (Barcelona: Roca Editorial, 2017), 38. 
rirnos a los elefantes, el bonobo - chimpancé pigmeo-, los delfines nariz de botella, las jirafas, todos ellos mantienen relaciones sexuales y afectivas de índole homosexual. Es más, existe un caso peculiar y es el de los berrendos o antílopes americanos, raza en la que entre el $66 \%$ y $75 \%$ de machos nunca se reproduce, dado que estos especímenes tienen sexo entre ellos y si establecen relaciones heterosexuales, lo hacen con las hembras cuya conducta sea primordialmente masculina.

b) En ese sentido, dado que la homosexualidad es una manifestación de la naturaleza, ha estado presente en la cultura peruana, al igual que en otras partes del mundo, desde tiempos remotos. Es decir, desde antes de la república, comprendiendo a las culturas preincaicas, al incanato y al virreinato.

Así, encontramos que diversos huacos de la cultura Mochica ${ }^{6}$, la misma que se remonta al período comprendido entre el año 300 a.C. hasta el 700 d.C., muestran actos homosexuales, los cuales eran retratados como actos normalizados pues formaba parte de su realidad el que haya parejas de mismo sexo.

Lo que respecta a la época incaica, también existieron manifestaciones homosexuales, tal como se desprende de los Comentarios reales de los Incas de Garcilaso de la Vega ${ }^{7}$. Cabe precisar que en determinados sectores del Tahuantinsuyo - zona central y sur del Imperio- esta práctica fue prohibida e, incluso, hasta castigada con la muerte. Mientras que, en la zona norte, era una conducta tolerada y, por qué no, hasta considerada como necesaria para dar placer a las tropas de los ejércitos incas ${ }^{8}$.

Sin embargo, esta situación varía completamente con el inicio de la conquista y la instauración del virreinato puesto que, como consecuencia de la influencia católica, toda práctica homosexual fue considerada como inmoral y debía ser reprimida con la intervención de la Santa Inquisición?. Ello generó que cualquier práctica de esta naturaleza se convierta en un hecho totalmente clandestino.

En consideración a los puntos expuestos, no cabe duda de que la homosexualidad, comprendiendo tanto a la masculina como a la femenina, es una manifestación de los integrantes de la comunidad LGBT+ y ha estado arraigada en la sociedad peruana desde, por lo menos, hace 20 siglos. En dicho sentido, y sin duda alguna, su presencia se mantendrá a lo largo del siglo XXI, salvo que en esta oportunidad se ha abierto una lucha constante por el reconocimiento de los derechos de esta población, garantizando así su desarrollo personal y el de sus proyectos de vida.

\section{LA REPÚBLICA DEL PERÚ Y LA COMUNI- DAD LGBT+ ANTES DEL SIGLO XXI}

La regulación sobre los derechos de la comu-

6. Juan José Candela Alva, «Los Huacos Eróticos En La Cultura Mochica», RPP en Archive.org, 5 de marzo de 2010, acceso el 18 de setiembre de 2020, https://web.archive.org/web/20150627075436/http:/radio.rpp.com.pe/peruanosenelexterior/los-huacos-eroticos-en-la-cultura-mochica/.

7. Liesder Mayea Rodríguez, «Un análisis de la representación y falta de representación del sujeto subalterno femenino u 'otro' en los Comentarios Reales Del Inca Garcilaso de La Vega», Revista Espéculo 46 (2010), http://webs. ucm.es/info/especulo/numero46/comreales.html.

8. "Cada tempo o adoratorio principal tiene un hombre, dos o más según el ídolo, los cuales andan vestidos como mujeres, desde el tiempo que eran niños; y hablaban como tales, y en su manera, traje y todo lo demás remedaban a las mujeres."

Pedro Cieza De León y Carmelo Sáenz De Santamaría, La Crónica del Perú. Las Guerras Civiles Peruanas, Tomo I, Capítulo LXIV (Madrid, Consejo Superior de Investigaciones Científicas: 1984), 90.

9. Luis Taylor Navas, «Evolución legislativa de los delitos sexuales», Anuario de Derecho Penal, 1999-2000, (2000): 2, http://perso.unifr.ch/derechopenal/assets/files/anuario/an_1999_15.pdf. 
nidad LGBT+ durante el siglo XIX no cambió en lo absoluto, en comparación a la existente durante el virreinato. Es más, el Código Penal de 1863 mantuvo la influencia española en lo que a regulación se refiere, por lo que su objeto fue la represión y sanción a quienes practiquen actos homosexuales.

Así, encontramos que el primer Código Penal peruano, el mismo que fue promulgado en $1863^{10}$, mediante su artículo 272 sancionaba la sodomía, lo que en ese entonces se identificaba como actos contra natura. Lo llamativo, hoy en día, es que la pena impuesta por este ilícito era la misma que se aplicaba para los casos de violación y estupro. Ello significa que, quienes sostenían relaciones sexuales homosexuales era equiparados con los violadores de esa época.

La estructura planteada fue reformulada 60 años después, cuando en $1924^{11}$ fue emitido el nuevo Código Penal, en el que primó la influencia suiza y trajo consigo cambios sustanciales, como la despenalización de los actos de sodomía y, de esa manera, desde hace casi 100 años, no existe sanción penal por tener relaciones sexuales con personas del mismo sexo.

Si bien las relaciones homosexuales no estuvieron penadas desde 1924, ello no implicó que existió una disposición normativa por la que tales actos sean respaldados. Se puede entender que ello cambió después de 55 años, cuando en 1979 entró en vigor una nueva constitución, por la que en su artículo 2 numeral 2, se dispuso que toda persona tiene derecho a la igualdad ante la ley sin discriminación alguna por razón de sexo. Si bien no es del todo certero, se podría entender que, además, este cuerpo normativo también comprendía a lo que hoy conocemos como la orientación sexual e identidad de género.
Sin perjuicio de ello, esta disposición normativa fue ampliada y precisada en la Constitución Política peruana de 1993, cuando en el artículo 2 numeral 2 establece que toda persona tiene derecho a la igualdad ante la ley sin discriminación alguna por "otra índole", lo que, ahora sí, puede comprender a los conceptos de orientación sexual e identidad de género.

Finalmente, y tomando en cuenta que el ser humano es el centro de la sociedad, así como de la legislación vigente, desde la perspectiva de los derechos humanos, tenemos que el artículo 1 de la Constitución Política de 1993 señala lo siguiente: "La defensa de la persona humana y el respeto de su dignidad son el fin supremo de la sociedad y del Estado". Con relación a este punto, el Tribunal Constitucional se ha pronunciado indicando qué debemos entender por dicha premisa:

"El Estado no sólo actuará respetando la autonomía del individuo y los derechos fundamentales como límites para su intervención (...), sino que deberá proporcionar, a su vez, los cauces mínimos para que el propio individuo pueda lograr el desarrollo de su personalidad y elección de sus planes de vida." ${ }^{\text {2 }}$

Tomando en cuenta lo dispuesto por el máximo intérprete de la Constitución Política de 1993, no es suficiente que exista un supuesto normativo por el cual se reconozca un derecho - como que la persona es el fin supremo de la sociedad y que esté libre de todo tipo de discriminación, lo que comprende a cualquier otra índole como la orientación sexual e identidad de género- sino que, además, el Estado debe brindar las herramientas mínimas para que cada individuo pueda desarrollarse conforme a su autodeterminación.

Teniendo ello en claro, sin perjuicio de la nor-
10. Ibíd., 11.
11. Loc. Cit.
12. Sentencia del Expediente $N^{\circ}$ 2016-2004-AA/TC, del 5 de octubre de 2004, fundamento 18. 
mativa infra constitucional ${ }^{13}$ promulgada hasta antes de que culmine el siglo $\mathrm{XX}$, procederemos a identificar los puntos en los cuales la comunidad LGBT+ carece de herramientas para desarrollar su personalidad y elegir, con la seguridad respectiva, como ejecutar sus planes de vida. Y, de la mano con ello, cuáles son los objetivos que debemos trazarnos como sociedad para el siglo XXI.

\section{LAS MEDIDAS NORMATIVAS ADOPTA- DAS POR LA REPÚBLICA DEL PERÚ DU- RANTE EL SIGLO XXI, PARA PROMOVER LA INCLUSIÓN DE LA COMUNIDAD LGBT+}

En el 2003 fue emitida la Ley 28044, Ley General de Educación, por la cual se señaló que los fundamentales principios entre los que se sustenta nuestro sistema educativo, encontramos el de equidad, la interculturalidad e inclusión. Así, respecto de este último concepto, encontramos que al sistema educativo se integran todas las personas sin que exista causa de discriminación, lo que comprende, inclusive, a las vinculadas a la orientación sexual e identidad de género.

En complemento al párrafo anterior, mediante la Resolución N² 281-2016-MINEDU se aprobó el currículo escolar 2017, por el cual se incluyó el enfoque de igualdad de género, buscando inculcar un esquema de igualdad de condiciones y posibilidades para los hombres y mujeres, a fin de que ejerzan sus derechos dentro de sus capacidades y oportunidades, promoviendo así su desarrollo personal.

Si bien esa disposición normativa fue cuestionada mediante una demanda de acción popular, planteada por el colectivo "Padres en Acción", este proceso concluyó con un pronunciamiento a favor del Ministerio de Educación, por el cual la Sala Constitucional y Social de la Corte Suprema de Justicia de la República concluyó que el Estado tiene el deber de erradicar los valores contrarios a la constitución, educando a los futuros ciudadanos en el respeto irrestricto a los derechos de todas las personas y que los enfoques, como el cuestionado, buscan una sociedad más igualitaria, por lo que no pueden ser sometidos a consulta.

Esto respaldó que la educación es un medio para desarrollar el respeto entre hombres y mujeres, así como de los miembros de la comunidad LGBT+.

Por otro lado, en el 2010 fue promulgada la Ley 29571, Código de Protección y Defensa del Consumidor, por la que se garantiza —-mediante su artículo 1.1.d- el derecho de todo consumidor a recibir un "trato justo y equitativo en toda transacción comercial y a no ser discriminador por motivo de origen, raza, sexo, idioma, religión, opinión, condición económica o de cualquier otra índole". Adicionalmente a ello, el artículo 38 de dicha norma establece la prohibición expresa de los actos por los que los consumidores sean víctimas de algún tipo de discriminación.

Finalmente, en enero de 2017, el Poder Ejecutivo emitió el Decreto Legislativo 1323, por el que modificó el Código Penal e incorporó, como categorías del delito de discriminación, a la orientación sexual e identidad de género. Asimismo, las establece como agravantes de la pena, con relación al artículo 46, inciso 2, literal d de dicho cuerpo normativo. Cabe precisar que el Poder Legislativo tuvo la intención de derogar ${ }^{14}$ el De-

13. Cabe precisar que el 26 de marzo de 1997 fue promulgada la Ley 26772, modificada por la Ley 27270, por la que se dispuso que las ofertas de empleo o requerimientos de personal no pueden contener requisitos que conlleven a un escenario de discriminación o vulneración a la igualdad de oportunidades o un trato diferenciado de las personas, basado en motivos de raza, sexo, religión, opinión, origen social, condición económica, estado civil, edad o de cualquier índole.

14. Ante ese escenario es que la Defensoría del Pueblo emitió el Oficio N 060-2017-DP, del 4 de abril de 2017, dirigido a la Presidencia de la Comisión de Constitución y Reglamento del Congreso de la República, por la que hizo hincapié en que la derogación de esta norma implicaría una infracción a las obligaciones convencionales asumidas por el Perú, así como un retroceso frente a la lucha contra la violencia. 
creto Legislativo 1323, alegando que el Poder Legislativo había excedido las facultades delegadas, situación que no se concretó.

En primer lugar, debemos advertir que la normativa vigente lo que regula es el derecho a la no discriminación, pero no brinda herramientas legales para el desarrollo de los planes de vida de los integrantes de la comunidad LGBT+.

Habiendo culminado con el análisis de la normativa vigente, antes de proponer los cambios que deben ser aplicados a nuestro ordenamiento, es necesario verificar cuál es la situación social que actualmente atraviesan los miembros de la comunidad LGBT+ en el Perú.

\section{LA SITUACIÓN SOCIAL ACTUAL DE LA CO- MUNIDAD LGBT+ EN EL PERÚ}

En junio de 2020 fue publicada la II Encuesta Nacional de Derechos Humanos de la Población LGBT $+{ }^{15}$, elaborada por el Ministerio de Justicia y Derechos Humanos e Ipsos, documento del que rescatamos las siguientes conclusiones:

a) Más de 1.7 millones de peruanos adultos se identifican con una orientación sexual no heterosexual.

b) El $71 \%$ de personas consideran que los homosexuales, bisexuales y transexuales son, por lo menos, discriminados.

c) La comunidad LGBT+ es la más discriminada en comparación a otros grupos minoritarios como las poblaciones indígenas, personas con discapacidad, las personas privadas de su libertad, etc.

d) El 37\% de encuestados no estaría dispuesto a contratar a una persona transexual en caso tuviese una empresa. El porcentaje sería del $30 \%$ si las personas a contratar fuesen homosexuales.

e) Existen estereotipos discriminatorios hacia la comunidad LGBT+, por lo que el $46 \%$ considera que una persona trans - transexual, transgénero y travesti- vive confundida; $y$, además, un $45 \%$ afirma que las personas se vuelven homosexuales por traumas en su infancia o por malas experiencias.

De la información identificada advertimos que, en primer lugar, la comunidad LGBT+ está constituida por una población representativa, la que merece ser protegida al igual que cualquier minoría. Por otro lado, existen claros problemas vinculados a la discriminación de los homosexuales y transgéneros, lo que deberá ser mitigado por medidas protectoras relevantes.

Así, en la siguiente sección identificaremos a profundidad los puntos en los cuales debemos trabajar para obtener una sociedad inclusiva para lo que resta del siglo XXI.

\section{LOS CAMBIOS NECESARIOS EN EL SIGLO XXI PARA SER INCLUSIVOS CON LA CO- MUNIDAD LGBT+}

Como hemos mencionado, el que la Constitución Política de 1993 regule expresamente el derecho a que los integrantes de la comunidad LGBT+ no sean discriminados no es suficiente para que desarrollen sus planes de vida en las mismas condiciones que los peruanos heterosexuales. Ello se debe a que estos últimos sí cuentan con las herramientas legales suficientes para cumplir con sus objetivos de vida como, por ejemplo, el matrimonio.

En ese escenario, enumeraremos y desarrollaremos de manera sucinta los puntos en los cuales el Estado debe poner manos a la obra y, adicionalmente, los que esperamos, como integran-

15. «Il Encuesta Nacional de Derechos Humanos», Ipsos, acceso el 18 de septiembre de 2020, https://www.ipsos.com/ es-pe/ii-encuesta-nacional-de-derechos-humanos. 
tes de la comunidad LGBT+, sean regulados en un mediano y largo plazo:

\section{Reconocimiento del matrimonio o unión civil entre parejas del mismo sexo.}

En el Perú solo está reconocido el matrimonio entre personas de diferente sexo, lo que excluye a las parejas homosexuales. Ello implica una limitación al acceso a los derechos hereditarios, por carecer de la calidad de cónyuges. Sin embargo, los efectos negativos son mayores a los que parecen.

Si una pareja homosexual decide desarrollar una vida en común y generar patrimonio común, cuando uno de ellos fallezca, el otro no podrá heredarlo, dado que se encuentra fuera de los supuestos de orden de prelación en materia sucesoria. Es más, al aplicar estos criterios, el patrimonio pasará a manos de la familia sanguínea del fallecido, dejando sin protección a su compañero.

Ello ocurrió en un conocido caso, en el que Armando y Alejandro habían compartido su vida por 26 años ${ }^{16}$. Los bienes adquiridos se encontraban a nombre de Alejandro y, cuando este falleció, sus hijos desalojaron a Armando del inmueble en el que vivía, pero que había sido adquirido con el patrimonio generado durante la convivencia de la pareja. Lamentablemente, no pudo ejercer herramienta legal alguna dado que su unión de hecho no se encontraba reconocida por nuestro ordenamiento.

Es importante mencionar que el reconocimiento del matrimonio o la unión de parejas del mismo sexo no solo se limita a los derechos sucesorios, sino que, además, comprende a la facultad para que uno de los integrantes de la pareja tome decisiones respecto del otro en situaciones de urgencia médica - así como que pueda visitar a su compañero si se encontrase internado- o para disponer de su cuerpo en caso fallezca.

Adicionalmente, la inexistencia de regulación también implica que, cualquiera de los integrantes en una relación homosexual no pueda ser beneficiado de los derechos prestacionales de salud ni a una pensión por viudez.

Debemos recalcar que, si bien el Poder Legislativo ha debatido la regulación sobre la relación de parejas del mismo sexo, nunca ha llegado a buen puerto. Para ello, usualmente argumentan que los integrantes de la comunidad LGBT+ pretenden la promulgación de normas que favorezcan a una minoría.

Sin embargo, no se toma en cuenta que, conforme lo señalado por el Tribunal Constitucional, el reconocimiento de un derecho constitucional no es suficiente, sino que, además, el Estado debe brindar las herramientas para que dicho derecho sea ejecutado efectivamente.

En el caso de los miembros de la comunidad LGBT+, si bien existe una prohibición a ser discriminados, no existe regulación por la que estas personas puedan ser consideradas como iguales en comparación a las heterosexuales, dado que no cuentan con las mismas herramientas para el desenvolvimiento de sus planes de vida. Por lo que, indirectamente, el Estado es el principal infractor de las disposiciones constitucionales, dado que brinda un trato diferenciado a la comunidad LGBT+, careciendo de una justificación objetiva para hacerlo.

2. Eliminar las limitaciones en el ejercicio del derecho a la identidad de las personas transgénero.

El principal problema que afrontan las perso-

16. «Armando Z. - Unión de Hecho», Incidencia Internacional PROMSEX, acceso el 18 de septiembre de 2020, https:// incidenciainternacional.promsex.org/armando-zorrilla-union-hecho/.

17. Ximena Salazar, Jana Villayzán, Alfonso Silva Santisteban y Carlos F. Cáceres, Las personas trans y la epidemia del VIH/Sida en el Perú: aspectos sociales y epidemiológicos. (Lima: IESSDEH, UPCH, ONUSIDA, AMFAR, 2010), 15. 
nas transexuales es que no existe un procedimiento definitivo y eficiente para que su sexo sea reconocido en sus documentos nacionales de identidad. Ello genera restricciones para el ejercicio de sus derechos como el acceso a la educación, al trabajo o a la salud.

Por otro lado, el hecho que exista una discordancia entre el aspecto físico y los documentos de identidad de las personas transgéneros también implica una exposición a situaciones de incomodidad, burlas o situaciones de confrontación en las veces que esta persona deba realizar un trámite o contratar un servicio ${ }^{17}$.

Prueba de lo descrito fue lo ocurrido durante el Estado Nacional de Emergencia Sanitaria, cuando el Gobierno determinó que los hombres saldrían a la calle determinados días de la semana mientras que las mujeres lo harían en los restantes. Omitiendo por completo el qué ocurriría con la población transexual. Así, como tomamos conocimiento a través de los medios de comunicación, cuando las personas transgéneros femeninas salieron a la calle en los días que les correspondían a las mujeres, eran retornadas a sus hogares dado que, para la Policía Nacional, según su documento de identidad eran identificadas como hombres ${ }^{18}$.

Los hechos descritos ocurrieron a lo largo de todo el Perú, lo cual suscrito que el mismo Presidente de la República brinde un mensaje a la nación, precisando que las reglas sobre restricción de tránsito serían aplicadas respetando la apariencia física de las personas, dejando de lado lo indicado en los documentos de identidad ${ }^{19}$.

Actualmente, en caso una persona transgénero requiera el cambio de sexo en su documento nacional de identidad, deberá iniciar un proceso judicial en el que, increíblemente, deben acreditar que viven y se sienten ${ }^{20}$ como hombres o mujeres, siendo el Juez quien, a su discrecionalidad, concluirá si corresponde declarar fundada la demanda.

En el congreso actual está pendiente la revisión y debate de la Ley de Identidad de Género, la misma que plantea la aprobación de un procedimiento administrativo por el que se facilitó la variación del sexo en el documento de identidad de las personas transexuales, así como en los demás archivos públicos — partida de nacimiento, etc.- a fin de facilitar que las personas transgéneros sean respetadas por su apariencia física y permitirle dejar su pasado de lado.

Mientras que ello no ocurra estaremos sometiendo a la población transgénero a situaciones discriminatorias de manera constante y sin justificación alguna.

\section{Eliminar las limitaciones al acceso a la sa- lud, al empleo y a la justicia.}

Como lo vimos al inicio, en el caso de Adriana, algunos centros médicos no atienden a las per-

18. Ariana Lira Delcore, «La Lucha de Las Mujeres Trans En Tiempos de Pandemia», El Comercio, 4 de diciembre de 2014, acceso el 18 de septiembre de 2020, https://especiales.elcomercio.pe/?q=especiales/mujeres-trans-entiempos-de-pandemia-ecpm/index.html.

19. Agencia EFE, «Gobierno Reitera Respeto a Identidad LGBTI En Medio de Medidas Contra El Coronavirus», Gestión, 3 de abril de 2020, acceso el 18 de septiembre, 2020, https://gestion.pe/peru/gobierno-reitera-respeto-a-identidad-Igbti-en-medio-de-medidas-contra-el-coronavirus-noticia/.

20. La Red Peruana TLGB y PROMSEX identificaron diversos problemas relativos a los procesos de cambio de nombre, entre ellos: "(i) la exigencia por algunos jueces de solicitar un certificado o examen que acredite la disforia de género (por considerar que lo transexual o transgénero es una patología), (ii) la exigencia de acreditar el inicio de la transformación del cuerpo como un requisito para el cambio de nombre y sexo; (iii) la demora en los procesos judiciales; y, (iv) la solicitud de diversos y variados requisitos para el cambio de nombre y sexo en función al criterio discrecional del juez a cargo del caso."

Liurka Otsuka Salinas y Soledad Arriagáda Barrera, Informe Anual sobre derechos humanos de personas trans, lesbianas, gays y bisexuales en el Perú 2011 (Lima, Red Peruana TLGB y PROMSEX, 2012): 35. 
sonas transexuales o a las homosexuales, ello sustentado en fundamentos discriminatorios. Situación que, indirectamente, genera un desincentivo para que estas personas sean atendidas, poniendo en riesgo su vida e integridad.

El Alto Comisionado de las Naciones Unidas para los Derechos Humanos ha señalado que:

“(...) las políticas, prácticas y actitudes discriminatorias de las instituciones y el personal sanitario repercuten negativamente en la calidad de los servicios de salud, disuaden a las personas de recurrir a esos servicios, y pueden llevar a que se deniegue la atención o a que no existan servicios que respondan a las necesidades sanitarias específicas de las personas LGBT e intersexuales". 21

En este escenario, se deben implementar medidas desde la perspectiva de la atención en salud, a fin de evitar que se generen supuestos de discriminación y, adicionalmente, la creación del procedimiento para registrar las denuncias que formulen los pacientes para trabajar en los puntos de mejora.

Por otro lado, en relación al acceso al trabajo, si bien existe una prohibición expresa ante la discriminación en este ámbito por cuestiones de orientación sexual e identidad de género, es necesario que se implementen herramientas o canales para la formulación de denuncias y, además, los remedios que serán empleados por las autoridades competentes para verificar estas faltas.

No debemos olvidar que, conforme a la II Encuesta Nacional de Derechos Humanos: Población LGBT, el $37 \%$ de encuestados no estaría dispuesto a contratar a una persona transexual en caso tuviese una empresa, mientras que el porcentaje sería del $30 \%$ si las personas a contratar fuesen homosexuales.
Estos datos nos permiten concluir que existe o podría existir un gran contenido discriminatorio en el ámbito laboral, el mismo que no ha sido identificado en la actualidad, por lo que debemos trabajar como sociedad en medios para identificar esta situación, erradicarla y promover un escenario de igualdad de oportunidades.

Finalmente, en lo que se refiere al acceso a la justicia, cuando nos encontramos ante casos penales en los cuales es necesaria la participación de la Policía Nacional durante la etapa de investigación, suele ocurrir que las autoridades incurren en supuestos que afectan a las víctimas de los delitos. Por ejemplo, los estereotipos que subyacen durante las investigaciones orientadas a identificar los motivos de los delitos cometidos en contra de las víctimas, el temor a las represalias, a la revictimización o el solo miedo a revelar su identidad sexual. ${ }^{22}$

En ese sentido, se deben implementar políticas para que las autoridades policiales, así como las fiscales empleen mecanismos para ser inclusivos y respetuosos respecto de las personas transgéneros $u$ homosexuales que han sido víctimas de delitos penales. Dado que, la falta de respeto a su padecimiento genera un desincentivo para que busquen la impartición de justicia.

Los tres puntos antes mencionados son los cruciales, en este momento, para generar una situación de equidad de oportunidades para los integrantes de la comunidad LGBT+ y los ciudadanos heterosexuales, que cuentan con un claro trato diferenciado y con herramientas legales para el desarrollo de sus planes de vida.

\section{CONCLUSIONES}

a) En primer lugar, los integrantes de la comunidad LGBT+ están cerca a los 2 millo-

21. Informe de la Oficina del Alto Comisionado de las Naciones Unidas para los Derechos Humanos sobre Discriminación y violencia contra las personas por motivos de orientación sexual e identidad de género (A/HRC/29/23), párrafo 50.

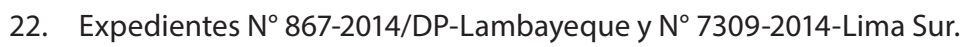


nes de ciudadanos peruanos, por lo que es una minoría representativa, cuya especial situación merece de una pronta regulación, a fin de erradicar la diferencia existente en comparación con los ciudadanos heterosexuales.

b) La homosexualidad, como manifestación de la comunidad LGBT+, es un tipo de orientación sexual que ha estado presente en nuestra historia desde hace más de 20 siglos, por lo que el otorgamiento de derechos que permitan su desarrollo no puede esperar más tiempo.

c) El derecho constitucional a no ser discriminados por cualquier otra índole - orientación sexual e identidad de género-impli$\mathrm{ca}$, adicionalmente, regular mecanismos para el desarrollo del plan de vida de las personas comprendidas en este universo.

d) Los principales puntos en los que debemos trabajar como sociedad son: (a) la regula- ción del matrimonio civil o unión de hecho, así como el otorgamiento de derechos sucesorios para las parejas homosexuales, (b) eliminar las limitaciones al ejercicio del derecho a la identidad de las personas transgéneros, promoviendo la identidad entre su aspecto físico con su documento de identidad; $y$, (c) garantizar el acceso a la salud pública, disminuir la discriminación en el acceso y sostenimiento del empleo; $y$, finalmente, establecer mecanismos para garantizar el acceso a la justicia de las personas de la comunidad LGBT+.

e) Habiendo identificado los puntos por cambiar, es necesario adoptar decisiones, como integrantes de la comunidad LGBT+, eligiendo mantener la situación vigente y vivir llenos de odio. O, promover un cambio a favor de todas las personas que lo necesiten o puedan necesitarlo en algún momento, para lo que debemos estar llenos de amor al momento de propulsar estas reformas. 